\title{
A ETIOLOGIA SOCIOECONÔMICA DA CÁRIE DENTAL E A PROMOÇÃO DA SAÚDE BUCAL EM CRIANÇAS E ADOLESCENTES - ÊNFASE À CIDADE DE BELÉM - PA
}

\author{
THE SOCIOECONOMIC ETIOLOGY OF DENTAL CARIES AND THE PROMOTION \\ OF ORAL HEALTH IN CHILDREN AND ADOLESCENTS - EMPHASIS ON THE CITY \\ OF BELÉM - PA
}

Eliane Paiva da Silva ${ }^{1}$

RESUMO: O Brasil caracteriza-se como o país com maior quantidade de cirurgiõesdentistas no mundo, no entanto, as disparidades sócio-econômicas geram uma grande discrepância na distribuição dos serviços odontológicos, o que representa um dos fatores para alto índice CPOD na região Norte. Crianças e adolescentes representam grupos de risco para o desenvolvimento da cárie dental, especialmente em regiões onde há menos atendimento odontológico, e mesmo pouca demanda de atendimento por parte da população, muitas vezes devido à falta da educação em saúde bucal, para compreender a importância da visita à consultórios odontológicos periodicamente. A educação em saúde bucal, também consta como um método de prevenção primária contra a cárie dental, portanto deve ser promovida a população, em especial para crianças e adolescentes que vivem em áreas de difícil acesso à serviços odontológico. Nesse sentido, o objetiva-se neste paper o estudo de fatores socioeconômicos como etiologia da cárie dental, bem como ações de promoção bucal como prevenção e mitigação da cárie dental, com ênfase a região de Belém - PA no Brasil.

Palavras-chave: Odontologia. Saúde coletiva. Prevenção primária. Educação em saúde bucal.

ABSTRACT: Brazil is characterized as the country with the largest number of dentists in the world, however, socioeconomic disparities generate a large discrepancy in the distribution of dental services, which represents one of the factors for a high CPOD index in the North region. Children and adolescents represent risk groups for the development of dental carie, especially in regions where there is less dental care, and even little demand for care by the population, often due to the lack of oral health education, to understand the importance of dental care and visit to dental offices periodically. Oral health education is also a method of primary prevention against dental caries, so it should be promoted to the population, especially to children and adolescents who live in areas with difficult access to dental care. In this sense, the objective of this paper is to study socioeconomic factors such as the etiology of dental caries, as well as oral health promotion actions such as prevention and mitigation of dental caries, with emphasis on the region of Belém - PA in Brazil.

Keywords: Dentistry. Collective health. Primary prevention. Oral health education.

\footnotetext{
IE-mail: eliane.financeiror6@gmail.com
} 


\section{INTRODUÇÃO}

O corrente processo de globalização que caracteriza a sociedade do século XXI modificou o modo de vida e o fluxo de informação de uma grande quantidade de pessoas no mundo, incluindo no Brasil. Não obstante, disparidades socioeconômicas e demográficas são observadas com frequência, e tais disparidades também estão presentes na área da saúde. $\mathrm{Na}$ odontologia, observa-se que os fatores socioeconômicos são sempre fatores que predispõe o acometimento por determinadas patologias, dentre essas a cárie dental. Crianças e adolescentes, caracterizando-se como grupo de risco a cárie dental, devem receber mais atenção odontológica. Nesse sentido, o objetiva-se neste paper o estudo de fatores socioeconômicos como etiologia da cárie dental, bem como ações de promoção bucal como prevenção e mitigação da cárie dental, com ênfase a região de Belém - PA. A metodologia utilizada foi pesquisa nas principais plataformas de saúde Biblioteca Virtual de Saúde (BVS), PubMed, visitas aos acervos de bibliotecas universitárias, apresentando com resultados nas línguas portuguesa e espanhola. Também foram realizados tabelas e cálculos estatísticos a partir de dados extraídos do Instituto Brasileiro de Geografia e Estatística (IBGE), Conselho Federal de Odontologia (CFO) e DATASUS.

\section{A etiologia socioeconômica da cárie dental e a promoção da saúde bucal em crianças e adolescentes da cidade de Belém - PA}

O Brasil caracteriza-se como o país que possui mais cirurgiões-dentistas no mundo, com cerca de $20 \%$ dos cirurgiões-dentistas do mundo habitando o Brasil, com mais de 330 mil profissionais da área. No entanto, observa-se que a distribuição dos mesmos no Brasil é desigual, portanto havendo regiões e populações brasileiras que recebem menor atendimento odontológico, seja de instituições públicas ou privadas. Isto pode ser observado através da análise da quantidade de estudantes que se formam em odontologia em cada região (Figura I).

De acordo com Galvão Filho, a cárie é descrita como um processo de "decomposição de tecido ou matéria orgânica; em odontologia consiste desintegração e dissolução de tecido ósseo ou dentário, tornando-o amolecido, descorado e poroso". Diversos tipos de cárie dental pode acometer a população, dependendo de sua localização no elemento dental, da idade e tipo de alimentação do indivíduo, bem como o uso de 
medicamentos e tratamentos (à exemplo de quimioterápicos e radioterapia), quantas vezes um determinado elemento dental foi acometido pela cárie dental, dentre outros fatores de análise.

Orellana Centeno et al (2021) relatam que a a cárie dentária, a perda dentária por cárie e as doenças periodontais são consideradas um dos maiores problemas de saúde mundiais, estimando-se que acometa cerca de 4 bilhões de pessoas no mundo. Os autores também relatam que as doenças bucais devem ser monitoradas não só do ponto de vista biomédico, mas também devem ter uma observação especial em relação ao ambiente onde o indivíduo se desenvolve, a consideração dos determinantes sociais e das iniquidades sociais em saúde, como uma parte importante para o desenvolvimento do processo saúdedoença, portanto possuindo grande relevância para observação.

Figura I - Concluintes do curso de odontologia em 2014.

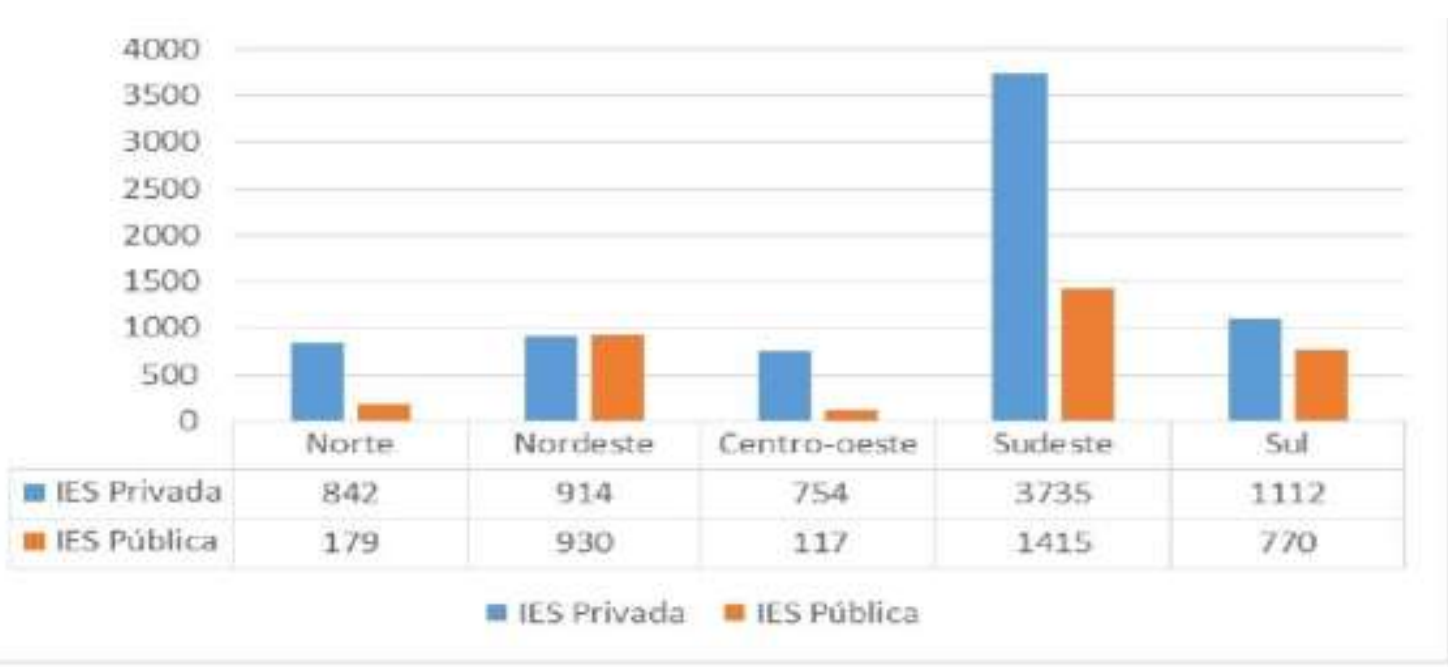

Fonte: GABRIEL, Mariana (2018)

Estima-se que 43.1\% do número total de cirurgiões-dentistas no Brasil trabalhem nas capitais dos estados (PEREIRA et al, 2018). Considerando o fato de que o Brasil possui 26 estados e suas respectivas capitais, e que o número total de cidades brasileiras é 5570, habitando nas capitais uma estimativa aproximada de 51 milhões dos aproximados 213 milhões de habitantes do país (Tabelas I,2,3,4 e 5), percebe-se um grande desequilíbrio, pois 43.1\% dos cirurgiões-dentistas são responsáveis por áreas que agrupam uma quantidade de aproximadamente apenas $23.8 \%$ da população brasileira, que torna-se sugestivo de um dos 
fatores de muitas populações no Brasil ainda não terem o acesso adequado a serviços de saúde bucal.

Tabela r: Número de habitantes das cidades capitais da região Norte.

\begin{tabular}{|l|l|l|l|}
\hline $\begin{array}{l}\text { Capitais da Região } \\
\text { Norte }\end{array}$ & $\begin{array}{l}\text { Número de } \\
\text { Habitantes da Capit } \\
\text { estimados em 2021. }\end{array}$ & $\begin{array}{l}\text { Número } \\
\text { Cirurgiões-Dentista } \\
\text { pelo estado respecti }\end{array}$ & $\begin{array}{l}\text { População Total } \\
\text { Estado respecti } \\
\text { estimados em 2021 }\end{array}$ \\
\hline Belém & 1.506.420 & 6852 & 8.777 .124 \\
\hline Boa Vista & 436.591 & 1042 & 652.713 \\
\hline Macapá & 522.357 & 1160 & 877.613 \\
\hline Manaus & 2.255 .903 & 5494 & 4.269 .995 \\
\hline Palmas & 313.349 & 2583 & 1.607 .363 \\
\hline Porto Velho & 548.952 & 2707 & 1.815 .278 \\
\hline Rio Branco & 419.452 & 1103 & 906.876 \\
\hline Total & 6.003 .024 & 20,941 & 18.906 .962 \\
\hline
\end{tabular}

Fonte: Instituto Brasileiro de Geografia e Estatística (IBGE) e Conselho Federal de Odontologia (CFO).

Tabela 2: Número de habitantes das cidades capitais da região Nordeste.

\begin{tabular}{|l|l|l|l|}
\hline $\begin{array}{l}\text { Capitais da Região } \\
\text { Nordeste }\end{array}$ & $\begin{array}{l}\text { Número de } \\
\text { Habitantes da Capit } \\
\text { Estimados em 2021. }\end{array}$ & $\begin{array}{l}\text { Número } \\
\text { Cirurgiões-Dentista } \\
\text { pelo estado respecti }\end{array}$ & $\begin{array}{l}\text { População Total } \\
\text { Estado respecti } \\
\text { estimados em 202I }\end{array}$ \\
\hline Aracaju & 672.614 & 2488 & 2.338 .474 \\
\hline Fortaleza & 2.703 .391 & 9375 & 9.240 .580 \\
\hline João Pessoa & 825.796 & 5937 & 4.059 .905 \\
\hline Maceió & 1.031 .597 & 3644 & 3.365 .351 \\
\hline Natal & 896.708 & 4631 & 3.560 .903 \\
\hline Recife & I.661.017 & 10774 & 9.674 .793 \\
\hline Salvador & 2.900 .319 & 16.264 & 14.985 .284 \\
\hline São Luís & I.115.932 & 5658 & 7.153 .262 \\
\hline
\end{tabular}




\begin{tabular}{|l|l|l|l|}
\hline Teresina & 871.126 & 3776 & 3.289 .290 \\
\hline Total & 12.678 .500 & 62.547 & $57,667,842$ \\
\hline
\end{tabular}

Fonte: Instituto Brasileiro de Geografia e Estatística (IBGE) e Conselho Federal de Odontologia (CFO).

Tabela 3: Número de habitantes das cidades capitais da região Sudeste.

\begin{tabular}{|l|l|l|l|}
\hline $\begin{array}{l}\text { Capitais da Região } \\
\text { Sudeste }\end{array}$ & $\begin{array}{l}\text { Número de } \\
\text { Habitantes da Capit } \\
\text { Estimados em 2021. }\end{array}$ & $\begin{array}{l}\text { Número de } \\
\text { Cirurgióes-Dentista } \\
\text { pelo estado respecti }\end{array}$ & $\begin{array}{l}\text { População Total } \\
\text { Estado respecti } \\
\text { estimados em 202I }\end{array}$ \\
\hline Belo Horizonte & 2.530 .701 & 42.442 & 21.411 .923 \\
\hline Rio de Janeiro & 6.775 .561 & 34.047 & 17.463 .349 \\
\hline São Paulo & 12.396 .372 & 103.745 & 46.649 .132 \\
\hline Vitória & 369.534 & 6955 & 4.108 .508 \\
\hline Total & 22.072 .168 & 187.189 & 89.632 .912 \\
\hline
\end{tabular}

Fonte: Instituto Brasileiro de Geografia e Estatística (IBGE) e Conselho Federal de Odontologia (CFO).

Tabela 4: Número de habitantes das cidades capitais da região Sul.

\begin{tabular}{|l|l|l|l|}
\hline Capitais da Região S & $\begin{array}{l}\text { Número de } \\
\text { Habitantes da Capit } \\
\text { Estimados em 202I. }\end{array}$ & $\begin{array}{l}\text { Número } \\
\text { Cirurgiões-Dentista } \\
\text { pelo estado respectiv }\end{array}$ & $\begin{array}{l}\text { População Total } \\
\text { Estado } \\
\text { estimados em 202I }\end{array}$ \\
\hline Curitiba & $\mathrm{I} .963 .726$ & 22.006 & $\mathrm{II} .597 .484$ \\
\hline Florianópolis & 516.524 & $\mathrm{I} 4.964$ & 7.338 .473 \\
\hline Porto Alegre & $\mathrm{I} .492 .530$ & 20.646 & $\mathrm{II} .466 .630$ \\
\hline Total & 3.972 .780 & 57.616 & 30.402 .587 \\
\hline
\end{tabular}

Fonte: Instituto Brasileiro de Geografia e Estatística (IBGE) e Conselho Federal de Odontologia (CFO)

Tabela 5: Número de habitantes das cidades capitais da região Centro-Oeste.

\begin{tabular}{|l|l|l|l|}
\hline $\begin{array}{l}\text { Capitais da Região } \\
\text { Centro-Oeste }\end{array}$ & $\begin{array}{l}\text { Número de } \\
\text { Habitantes da Capit } \\
\text { Estimados em 2021. }\end{array}$ & $\begin{array}{l}\text { Número } \\
\text { Cirurgiões-Dentista } \\
\text { pelo estado respectiv }\end{array}$ & $\begin{array}{l}\text { População Total } \\
\text { Estado respecti } \\
\text { estimados em 202I }\end{array}$ \\
\hline Brasília & 3.094 .325 & 8536 & 3.094 .325 \\
\hline Goiânia & $\mathrm{I} .555 .626$ & 12.912 & 7.206 .589 \\
\hline
\end{tabular}




\begin{tabular}{|l|l|l|l|}
\hline Campo Grade & 916.001 & 4933 & 2.839 .188 \\
\hline Cuiabá & 623.614 & 6136 & 3.567 .234 \\
\hline Total & 6.189 .566 & 32.517 & 16.707 .336 \\
\hline
\end{tabular}

Fonte: Instituto Brasileiro de Geografia e Estatística (IBGE) e Conselho Federal de Odontologia (CFO)

Analisando-se os dados obtidos da demografia aproximada e da quantidade de cirurgiões-dentistas por região, observa-se que a região Norte e Nordeste possuem a menor quantidade de cirurgiões-dentistas por habitante, sendo na região Norte aproximadamente I cirurgião-dentista para cada 902,8 habitantes em 202I. Comparando-se com a média do país que se apresenta em I Cirurgião-Dentista para cada 591,2 habitantes, observa-se a discrepância das regiões Norte e Nordeste em relação ao país. Ao relacionar-se com os dados de Pereira et al (2018), no qual 43,1\% dos Cirurgiões-Dentistas estão nas capitais, a discrepância de cirurgiões-dentistas por habitante é sugestiva de ser muito maior em comunidades mais afastadas das capitais. Tais fatos podem também serem sugestivos de a região Norte ser a única que apresentou aumento no índice CPO-D no ano de 2010 (Tabela 6).

Tabela 6: Índice CPO-D feitos entre 1986 e 2010.

\begin{tabular}{|l|l|l|l|l|}
\hline & 1986 & 1996 & 2003 & 2010 \\
\hline Norte & 7,5 & 4,3 & $3, \mathrm{I}$ & 3,2 \\
\hline Nordeste & 6,9 & 2,9 & 3,2 & 2,7 \\
\hline Centro-Oeste & 8,5 & 2,9 & 3,2 & 2,6 \\
\hline Sudeste & 6,0 & $2, \mathrm{I}$ & 2,3 & 1,7 \\
\hline Sul & 6,3 & 2,4 & 2,3 & 2,0 \\
\hline Brasil & 6,7 & $3, \mathrm{I}$ & 2,8 & $2, \mathrm{I}$ \\
\hline
\end{tabular}

Fonte: Departamento de Informática do SUS (DATASUS)

O flúor é um importante elemento anticariogênico, o mais utilizado em odontologia ao combate a patologia da cárie dental. Desde a primeira metade do século XX 
o flúor vem sendo estudado dentro da odontologia, e graças aos estudos de diversos cientistas a respeito desse elemento, em 1945 houve a primeira fluoretação bem sucedida em Grand Rapids, Michigan, e a partir da segunda metade do século XX diversos países passaram a fluoretar as águas de abastecimento como medida de prevenção à cárie dental. Deve-se ressaltar que a fluoretação das águas precisa ser frequentemente controlada e avaliada, pois abaixo da quantidade recomendada de partículas por milhão o flúor é ineficaz, bem como acima da quantidade, acarreta prejuízos à saúde, como a fluorose dental. Conforme Rodrigues et al (202r), na cidade Belém no Pará a fluoretação artificial iniciou-se em 1985 e no ano de 1996 82\% da população já recebia água fluoretada, não obstante, em uma análise das águas de Belém realizada em 2003, 78\% das águas de abastecimento apresentavam níveis de flúor abaixo do recomendado.

De acordo com Ortiz (2018), a cárie dental também possui impactos sociais, que envolvem a autopercepção física e ambiental das crianças e adolescentes, assim como seus responsáveis, gerando impactos na qualidade de vida, com a piora do desempenho escolar, perda de peso, irritabilidade e diminuição da autoestima. Em contrapartida, isso também afeta os pais e responsáveis, que muitas vezes têm despesas com tratamentos, faltas ao trabalho para acompanhar os filhos à consulta com cirurgião-dentista, além de possíveis sentimentos de culpa, portanto afetando o bem-estar social e familiar.

Um importante aspecto da promoção à saúde e combate à doença cárie dental é a adequação do meio bucal. Lima et al (2020) descrevem a adequação do meio bucal do paciente como o "controle dos diversos fatores relacionados à cárie dentária, visando o equilíbrio biológico do meio bucal, o qual é imprescindível para orientar o tratamento sob o prisma da promoção da saúde." Os autores também relatam que esta fase do tratamento "objetiva reconduzir o processo da desmineralização ao equilíbrio dinâmico, favorecendo os episódios de remineralização pelo controle dos fatores da doença, o que pode ser alcançado através da instituição de medidas relativamente simples" das quais foi-se destacado o controle de placa; a utilização de fluoretos; orientação de higiene bucal, controle da dieta, identificação condições de risco e o selamento das cavidades.

É necessário ressaltar a importância da educação em saúde bucal, e o que diz respeito a ampliação desta. Guedes II (2021), descreve a importância do ensino da saúde 
bucal e a necessidade da implementação desta na grade curricular de estudantes do ensino fundamental II descrevendo:

A saúde é um dos temas mais importantes de serem ensinados no ensino fundamental II. É neste momento que o estudante está aprendendo mais sobre o corpo humano e é essencial que o aluno perceba a aplicabilidade do conteúdo lecionado para o seu dia-a-dia. Neste contexto, a saúde bucal tem grande importância de ser ensinada ao estudante, proporcionando o seu bem-estar físico, social e mental, melhorando sua qualidade de vida, pois através da educação em saúde bucal há maior chance da prevenção de diversas patologias, das quais destacam-se a cárie dental e doenças periodontais."

A adolescência é período de risco aumentado à cárie dentária, decorrente da busca do equilíbrio físico-psíquico-social, e demonstração de comportamentos extremos, ora positivos e ora negativos em relação à saúde. Adolescentes não possuem os cuidados dispensados às crianças e tão quanto desfrutarem da assistência direcionada aos adultos, o que induz a aquisição de fatores determinantes relacionados à cárie dental; dentre esses o pouco controle da placa e cuidados com a higienização bucal, novos hábitos alimentares (preferência por lanches rápidos, salgadinhos, chicletes e refrigerantes), além de hábitos adquiridos que potencializam o comprometimento da saúde bucal (Piercing, álcool, tabaco), maior exposição ao traumatismo buco-dental através de brincadeiras e desporto (LIMA et al, 2020). Guedes II (2020) e Lima et al (2020), chamam atenção a um maior empenho na educação e orientação em saúde bucal como prevenção à cárie dental.

No território da Grande Belém que compreende 7 municípios, existem 50 ilhas nos rios Guamá e Pará, compreendendo cerca de 40\% do território da Grande Belém, com uma população aproximada de 8260 pessoas no ano 2000 (MEIRELLE FILHO, 2014). Sabese que a população ribeirinha possui baixa renda e pouca instrução escolar, ambos são importantes fatores para um alto risco de cárie, especialmente em crianças e adolescentes. Torna-se muito importante a promoção e prevenção da saúde bucal em todos os níveis para tais populações. Campanhas, ações sociais, e variados métodos de orientação e educação em saúde bucal devem ser estimulados, tanto a partir de programas do governo como o Brasil Sorridente, como pelos próprios cirurgiões-dentistas. Em ações sociais em tais comunidades, no que diz respeito à odontopediatria e odontohebiatria, deve-se dar foco à adequação do meio bucal em um primeiro momento. Dadas as dificuldades para atendimento nesses territórios, os procedimentos dentais como selantes de fóssulas e 
fissuras, remoção de tecido cariado com escavador de dentina, tratamento restaurador atraumático e aplicação tópica de flúor são de grande valia.

\section{CONCLUSÃO}

A etiologia socioeconômica é um fator determinante no contexto da cárie dental. Observa-se que a desigualdade econômica apresenta efeito direto sobre a saúde das populações de regiões com menor desenvolvimento econômico, as quais se incluem a cidade de Belém e regiões associadas a essa. É necessário mais ações de prevenção contra a cárie dental, em especial a promoção da educação da saúde bucal em crianças e adolescentes, pois a educação é uma peça fundamental para a prevenção de diversas doenças bucais, em especial a cárie dental. Programas governamentais como a fluoretação de águas de abastecimento devem receber maior atenção para a eficácia destes, bem como ampliação para abranger maiores parcelas da população, gradativamente até alcançar o total da população.

\section{REFERÊNCIAS}

CONSElHO FEDERAL DE ODONTOLOGIA. Quantidade Geral de Profissionais e

Entidades Ativas. 2021. Disponível em https://website.cfo.org.br/estatisticas/quantidadegeral-de-entidades-e-profissionais-ativos/ Acesso em 21 de Setembro de 202I.

DEPARTAMENTO DE INFORMÁTICA DO SUS - DATASUS. Fichas de Qualificação da RIPSA - 2012, Fatores de Risco e de Proteção, G.17: banco de dados. Disponível em: http://fichas.ripsa.org.br/2012/g-17/?l=pt_B Acesso em 2i de Setembro de 2021.

GABRIEL, Mariana. Distribuição geográfica dos Cirurgiões-Dentistas: percepção e motivação para a migração. Tese (Doutorado em Ciências Odontológicas). Programa de Pós-Graduação em Ciências Odontológicas da Universidade de São Paulo. p.I55. São Paulo, 2016. Disponível em https://www.teses.usp.br/teses/disponiveis/23/23148/tde07062017-162536/publico/MarianaGabrielVersaoOriginal.pdf Acesso em o9 de Setembro de 2021.

GALVÃO FILHO, S. Dicionário odonto-médico: inglês - português. 5 ed. São Paulo : Santos, 20II. ISBN 978-85-7288-775-5.

GUEDES II, Aureliano da Silva. EDUCAÇÃO EM SAÚdE PARA ESTUDANTES DO ENSINO FUNDAMENTAL II: ÊNFASE A SAÚDE BUCAL. Trabalho de Conclusão de Curso (Formação Pedagógica em Ciências Biológicas). Faculdades Integradas de Ariquemes. Ariquemes - RO, p.I6. 2021. 
IBGE - INSTITUTO BRASILEIRO DE GEOGRAFIA E ESTATÍSTICA . Estimativa do Censo Brasileiro de 2021. Rio de Janeiro: IBGE, 2021. Disponível em https://cidades.ibge.gov.br/ Acesso em 20 de Setembro de 202I.

LIMA, Maria Germana Galvão Correia; et al. A adequação do meio bucal para o controle da cárie dental em adolescentes. Brazilian Journal of Health Review., Curitiba, v. 3, n. 3, p.5122-5138 may/jun. 2020. Disponível em https://www.brazilianjournals.com/index.php/BJHR/article/view/Io622 Acesso em I4 de Setembro de 202I

MEIRELles FILHO, João. Belém Ribeirinha - Marco Contextual. Instituto Peabiru. Belém, 2014. Disponível em https://www.academia.edu/9782238/Bel\%C3\%A9m_Ribeirinha_Marco_Contextual Acesso em 18 de Setembro de 2021.

ORELLANA CENTENO, José Eduardo; GUERRERO SOTELO, Roxana Nayeli; MORALES CASTILLO, Verónica. La relación entre índices socioeconómicos y la salud oral. Revista de Salud Pública 25 (I), 86-94. 2021. Disponível em https://doi.org/10.31052/1853.1180.v25.ni.29809 Acesso em I2 de Setembro de 2021.

ORTIZ, Adriana Santos. INFLUÊNCIA DAS INIQUIDADES SOCIOECONÔMICAS NA OCORRÊNCIA DE CÁRIE DENTAL EM ADOLESCENTES. Dissertação (Mestrado em Ciências Odontológicas). Programa de Pós-Graduação em Ciências Odontológicas. Universidade Federal de Santa Maria. Santa Maria-RS, p. 64. 2018.

PEREIRA, Fabio; MENDONÇA, Iranilda A. de; WERNECK, Renata I.; et al. Human Development Index, Ratio of Dentists and Inhabitants, and the Decayed, Missing or Filled Teeth Index in Large Cities. The Journal of Contemporary Dental Practice, November 2018;19(II):1363-1369. Disponível em https://www.thejcdp.com/abstractArticleContentBrowse/JCDP/19/19/11/15155/abstractA rticle/Article Acesso em 20 de Agosto de 2021.

RODRIGUES, Ayrton Breno Teixeira; et al. O panorama da fluoretação das águas de abastecimento público da cidade de Belém, estado do Pará, Brasil. Revista Pan-Amazônica de Saúde. n.12. Jul/2021. Disponível em http://dx.doi.org/I0.5123/s2176-6223202100708 Acesso em i7 de Setembro de 202I. 